\title{
206.
}

\section{NOTE ON A THEOREM RELATING TO THE RECTANGULAR HYPERBOLA.}

[From the Philosophical Magazine, vol. XIII. (1857), p. 423.]

THE following theorem is given in a slightly different form by Brianchon and Poncelet, Gergonne, vol. XI. [1820], p. 205, viz. Any conic whatever which passes through the three angles of a triangle and the point of intersection of the perpendiculars let fall from the angles of the triangle upon the opposite sides is a rectangular hyperbola; and there is an elegant demonstration depending on the properties of the inscribed hexagon. The theorem is, however, a particular case of the following: viz. "Any conic whatever which passes through the four points of intersection of two rectangular hyperbolas is a rectangular hyperbola." And this, again, is a particular case of the following: viz. If there be a conic $\Omega$ and a line $P$, then considering any two conics $U, V$ such that the points of intersection of $P, U$ are harmonics in respect to the points of intersection of $P, \Omega$, and the points of $P, V$ are also harmonics in respect to the points of intersection of $P, \Omega$, then any conic whatever $W$ which passes through the four points of intersection of $U, V$ will have the like property, viz. the points of intersection of $P, W$ will be harmonics in respect of the points of intersection of $P, \Omega$; a theorem which is an immediate consequence of the theorem that three conics which intersect in the same four points are intersected by any line whatever in six points which are in involution.

2, Stone Buildings, W.C., April 23, 1857. 